\title{
LEP Experiments Produce First Major Results
}

On 13 November 1989, LEP was formally inaugurated in the presence of H.M. King Carl XVI Gustaf of Sweden, H.R.H. Princess Margriet of the Netherlands, H.R.H. Prince Harald of Norway, the Presidents of the two countries through which LEP runs, François Mitterrand of France and Jean-Pascal Delamuraz of Switzerland, the President of EPS and distinguished representatives of the governments of the States participating in the CERN programme and the world physics community.

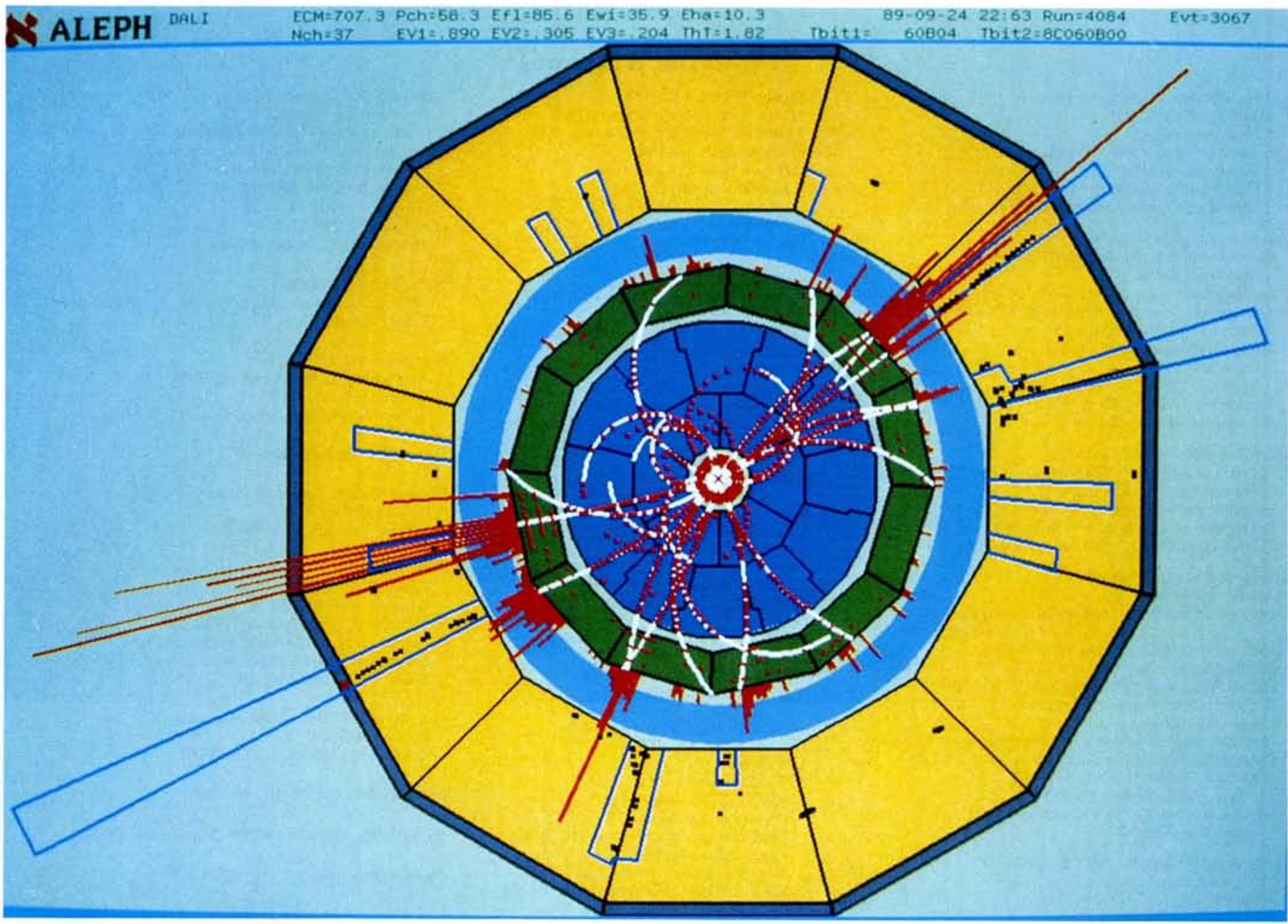

Computer visualization of a LEP event. (The actual reconstruction of events is performied in three dimensions and provides several hundred kilobytes of data of which only the main features can be represented graphically on this scale.) The detector shown here (Aleph) is typical in that it consists of a series of sub-detectors arranged concentrically about the beam pipe (the black spot in the middle) where a collision between an electron and a positron yields the event depicted here. The innermost sub-detector, shown in red and white, gives the first points on the trajectories of charged particles, too detailed to be represented here. Next out from the centre, red points and white tracks on blue background, show the charged particle trajectories bending in the $1.5 \mathrm{~T}$ magnetic field, and detected in the main tracking chamber, $4 \mathrm{~m}$ in diameter. Outside this in green is the electromagnetic calorimeter, showing in white the trajectories and as red histograms the energy losses of both charged and neutral particles. Further out are the superconducting magnet (light blue) and the hadron calorimeter (yellow), which is contained in the iron return yoke of the magnet. The open histograms indicate the particles' energy loss; hadrons normally stop very suddenly in the iron. In this event, one muon in the lower left traverses all the iron and is detected in a muon chamber, the thin outer shell, and another likely muon on the upper right side does not quite get out.
In spite of the unprecedented size and complexity of the LEP machine and the four LEP experiments (see Europhysics News June 1989 issue), the first physics results are already coming out. Less than ninety days after the first electronpositron beams made a full turn of the $27 \mathrm{~km}$ long tunnel, all four experimental collaborations had reported physics results for publication, and several additional papers are in preparation.

At the time of writing (1 Nov.) the four teams have already observed over $20000 \mathrm{Z}^{\circ}$ events, and are busy collecting more. Current running conditions provide about $100 \mathrm{Z}^{\circ}$ per hour to each experiment after successful fills, storage lasting about six to eight hours in the rings. This can be compared with less than 100 identifiable $Z^{\circ}$ events per year produced at the big hadron colliders of CERN and Fermilab, or the current production rate of about 100 events per month in the Mark II detector at the Stanford Linear Collider in California.

The first physics results are much improved measurements of the $Z^{\circ}$ mass $(91.10 \pm 0.06 \mathrm{GeV})$, width, and decay rate into hadrons, all of which can be combined to exclude, to a very high confidence level, the existence of a new type of light neutrino in addition to the three known species. This in turn means it is unlikely that there are any new quarks or leptons beyond the three known families of each. Further important results include the pinning down of the details of interactions between quarks, described by quantum chromodynamics or QCD.

The major disappointment is the absence so far of any unexpected behaviour; perhaps high energy physics is finally becoming a mature science. 